\title{
Evaluación chilena del Inventario de Comportamientos de la Pareja que Provocan Celos
}

\section{Chilean assessment of the Inventory of Jealousy-Evoking Partner Behaviors}

\author{
Ana María Fernández , Paula Pavez y Michele Dufeyc \\ ${ }^{\mathrm{a}}$ Universidad de Santiago de Chile, Santiago, Chile ${ }^{\mathrm{b}}$ Universidad Autónoma de Chile, Santiago, Chile \\ cUniversidad Diego Portales, Santiago, Chile
}

\section{Resumen}

El presente estudio muestra la adaptación al contexto local del Inventario de Comportamientos de la Pareja que Provocan Celos (ICPC) creado en Holanda por Dijkstra, Barelds y Groothof (2010). Un pilotaje del inventario arrojó alta consistencia interna y estabilidad test-retest. Se administró el cuestionario a un grupo de 366 personas y 31 participantes completaron el retest. Un análisis factorial exploratorio permitió diferenciar las particularidades del instrumento en su configuración original y el contexto local, convergiendo en tres dimensiones: comportamiento infiel, comportamiento sospechoso y atracción mediática. Los resultados indicaron similares valores de consistencia interna para las dimensiones locales y las dimensiones de la escala original, así como los mismos patrones de correlación de las dimensiones entre sí; al igual que diferencias sexuales esperables desde el marco evolucionario, siendo las mujeres quienes responden con mayores valores en dos de las dimensiones de este constructo, reflejando una fuerte reacción basal a las situaciones que amenazan una relación valorada.

Palabras clave: reactividad a los celos, diferencias sexuales, psicología evolucionaria.

\begin{abstract}
The current investigation reports the local adaptation of the Inventory of JealousyEvoking Partner Behaviors (IJEPB) which was created in Netherlands by Dijkstra, Barelds \& Groothof (2010). A pilot study showed high internal consistency and test-restest reliability. The questionnaire was completed by 366 people and 31 of them completed it again on the retest. An exploratory factor analysis allowed for differentiating the particularities of the instrument in the local context from its original configuration. The data resulted in three-dimensions: unfaithful behavior, suspicious behavior, and media attraction. Other results showed values of internal consistency comparable to the Dutch dimensions of the scale, as well as similar correlations among the factors. Sex-differences in jealousy reactivity conformed to evolutionary precepts, with women declaring higher responses on two of the dimensions of the construct, possibly reflecting a stronger overall baseline reaction to situations that threaten a valued relationship.
\end{abstract}

Keywords: jealousy reactivity, sex differences, evolutionary psychology.

Esta investigación contó con el financiamiento del proyecto Fondecyt regular \#1140234 y Fondecyt de iniciación \#11110439.

Contacto: A. M. Fernández. Escuela de Psicología. Universidad de Santiago de Chile. Avenida Ecuador 3650,3er Piso.Santiago. ana.fernandez@usach.cl.

Cómo citar este artículo:

Fernández, A. M., Pavez, P. y Dufey, M. (2014). Evaluación chilena del Inventario de Comportamientos de la Pareja que Provocan Celos. Revista de Psicología, 23(2), 45-55.

http://dx.doi.org/10.5354/0719-0581.2014.36147 


\section{Introducción}

Los sentimientos de celos son generados por la amenaza real o percibida de pérdida de una relación valiosa, pudiendo ser esta provocada por un rival real o imaginario, que supone desviar la atención de la pareja (Buss, Larsen, Westen y Semmelroth, 1992; Dijkstra, Barelds y Groothof, 2010; Dijkstra y Buunk, 2002). Los individuos que experimentan celos comparan sus características con las del rival y cuando observan que este supera sus propias cualidades, pueden percibirlo como una amenaza para la relación $\mathrm{y}$, en consecuencia, evocar sentimientos de celos (Dijkstra y Buunk, 2002).

Los teóricos de los celos románticos indican que esta conducta implica una compleja mezcla de emociones, pensamientos y comportamientos como, por ejemplo, sentimientos de ansiedad, insatisfacción y problemas de relación, y en algunos casos esto conlleva al abuso físico y/o verbal (Elphinston, Feeney y Noller, 2011).

Desde hace algunos años, ha existido un nexo ampliamente estudiado y desarrollado teóricamente en EEUU y Europa acerca de tres disciplinas que estudian la estructura de la mente humana: las neurociencias, la biología evolutiva y la psicología cognitiva, dando origen a la psicología evolucionaria (Barkow, Cosmides y Tooby, 1992; Buss, 2005, 2009, 2011; Cosmides y Tooby, 1999, 2013).

Esta aproximación científica es un paradigma integrador, que pretende reconstruir los problemas a los que se enfrentaron nuestros antepasados en su entorno primitivo, para comprender los mecanismos psicológicos que desarrollaron para resolverlos (Sznycer, Tooby y Cosmides, 2011). Incluye además, como medio generador de mecanismos psicológicos o programas mentales, la teoría de la evolución de Darwin (1859) para explicar a través de los principios de selección natural y sexual, las características universales del comportamiento humano, considerando las diferencias y similitudes del contexto ancestral y el presente (Barkow et al., 1992).

La psicología evolucionaria establece que el comportamiento humano estaría motivado por una función adaptativa que se transmite de generación en generación $y$, a partir de esta, explica las raíces comunes de nuestro comportamiento ancestral, mostrándonos cómo este permanece hasta la actualidad en todas las culturas del mundo, pese a su variedad y dispersión (Sznycer et al., 2011). De igual forma, esta perspectiva sostiene y evidencia que la mente y el comportamiento humano son susceptibles a variables del entorno, como cualquier mecanismo adaptativo lo es (Cosmides y Tooby, 1992; Darwin, 1859). Finalmente, propone que la mente humana es producto de la evolución (Buss, 1995, 2011; Cosmides y Tooby, 1992, 1999, 2013), y con ello lo son también los mecanismos psicológicos que la conforman, como las emociones y las habilidades cognitivas.

Desde la perspectiva evolucionaria, se reconoce que la mente humana está diseñada para resolver problemas asociados a la supervivencia y a la reproducción, y que nuestra especie ha evolucionado gracias a la existencia de estos mecanismos adaptativos, incluyendo la selección de una pareja que garantice el éxito en la transmisión de los propios genes (Buss, 2013a; Cosmides y Tooby, 2013; Maner y Shackelford, 2008). De esta forma, los celos en el contexto de una relación reproductiva, son considerados una respuesta adaptativa cuya finalidad sería proteger una pareja valorada y prevenir que un rival desvíe la atención de esta (Buss, 2005).

Buss et al. (1992) y Buss et al., (1999) han observado, estimado y analizado las diferencias entre hombres y mujeres en su experiencia de celos, según el tipo de infidelidad en que pudiese incurrir la pareja. Ellos llegan a la conclusión que los hombres se sienten más agraviados por una infidelidad sexual de sus compañeras que las mujeres, quienes conceden mayor preocupación a una infidelidad de tipo emocional. Este punto de vista es coincidente con las observaciones preliminares de Buss (1989) sobre diferencias entre hombres y mujeres al valorar los atributos que resultan atractivos en un compañero (de Miguel y Buss, 2011). Los resultados transculturales y metaanálisis revelan que las diferencias sexuales en los celos, responden a las preferencias universales de hombres, quienes en mayor medida que las mujeres consideran el atractivo físico y la exclusividad se- 
xual de la pareja como un factor esencial a la hora de seleccionar una pareja. En contraste, para las mujeres se ha encontrado una mayor molestia por la pérdida de la exclusividad romántica, que es coincidente con su alta atracción por el status y el dominio social que puede proporcionar su compañero (Buss, 2009; Buunk y Dijkstra, 2001; Dijkstra y Buunk, 2002).

De acuerdo con Dijkstra et al. (2010) el estudio de las situaciones que evocan celos dentro de una díada romántica se torna relevante por varias razones como, por ejemplo, el hecho que la infidelidad y los sentimientos de celos derivados de esta son difíciles de tratar y los profesionales a menudo no se sienten preparados para este tipo de intervención. Por otro lado, tal como lo han planteado autores evolucionarios, los celos son una respuesta esperable y no necesariamente ajena a las relaciones de pareja saludables (cf. Buss, 2005; Dunbar, 2012; Fisher, 1996).

Igualmente, los celos son a menudo entendidos como una conducta patológica de parte de una pareja que podría tener baja autoestima, cogniciones irracionales o algún tipo de trastorno de personalidad, aunque también estos pueden ser causados por una pareja que constantemente evoca y fortalece involuntariamente la conducta de celos del otro (Buunk, 1997; Dijkstra et al., 2010).

Sin embargo, y según la perspectiva de la psicología evolucionaria, los celos dentro de una relación de pareja son una emoción esperable, que motiva la protección de la relación, lo cual resulta adaptativo al buscar mantener una relación potencialmente reproductiva (Fernández, 2009). Similarmente, Buss (2013b) ha explicitado la idea que los celos románticos serían una emoción básica fundamental para la evolución de la especie humana, argumento desarrollado en el trabajo experimental con respuestas emocionales ante la imaginería de infidelidad de Fernández (2012), en nuestro contexto nacional.

Por tanto, en la presente investigación se considera que el disponer de un inventario local para conocer el grado en que diversos comportamientos de provocación de celos afectan a las personas, puede ayudar a nuestra disciplina a estimar, conocer, analizar y avanzar en la comprensión de esta respuesta emocional, en el contexto de las relaciones románticas. Asimismo, la adaptación del Inventario de Comportamientos de la Pareja que Provocan Celos (ICPC), puede facilitar la investigación de esta emoción en conjunto con otras características de las relaciones de pareja, variables de personalidad, etcétera, al mismo tiempo que puede motivar la investigación clínica orientada a que las parejas y los terapeutas, por ejemplo, puedan identificar situaciones, patrones de comportamiento y desarrollo de tratamientos adecuados para los celos exacerbados o disfuncionales.

Por consiguiente, el estudio buscó adaptar el ICPC a nuestro país, realizando su traducción y adaptación al lenguaje local, para luego estimar su fiabilidad y validez de la misma forma que se conceptualizó su versión original en inglés (Dijistra et al., 2010).

\section{Participantes}

\section{Método}

Un total de 366 personas heterosexuales (28\% hombres) entre 18 y 63 años de edad $(M=$ 27.91, $D E=10.09)$ participaron en el estudio. Un $66 \%$ de la muestra indicó tener una pareja al momento del estudio ( $25 \%$ de los hombres y $75 \%$ de las mujeres). La muestra fue de tipo accidental (Hernández, Fernández-Collado y Baptista, 2010), ya que solo se intentó incorporar la mayor cantidad de participantes de ambos sexos posible, en un período de ocho meses de aplicación del estudio, siendo la meta alcanzar al menos cinco personas por ítem del inventario adaptado, según el criterio clásico de Nunnally y Bernstein (1994).

\section{Procedimiento}

Las personas fueron invitadas a participar en un estudio online mediante un vínculo por correo electrónico, a través de letreros en la cafetería de las instituciones a las cuales pertenecen los autores y por las redes sociales. Una vez iniciada la investigación, los participantes debieron leer y descargar el consentimiento informado aprobado por la institución patroci- 
nante, antes de poder responder el ICPC y las preguntas demográficas incluidas en el estudio (edad, nivel socioeconómico, nivel de estudios y apreciación global de qué tan celosa es la persona; Buunk, Park, Zurriaga, Klavina y Massar, 2008).

El ICPC fue traducido al español mediante el método de back-translation (Muñiz, Elosua y Hambleton, 2013; Triandis y Berry, 1980), el cual consiste en la traducción al español del instrumento en inglés por un psicólogo bilingüe y, posteriormente, en la traducción de vuelta al inglés del instrumento preliminar en español por parte de otro psicólogo bilingüe. Finalmente, dentro del equipo de investigación se compararon ambas versiones del instrumento resaltando las diferencias de significado, y se repitió el proceso de traducción reiterativamente hasta que no se encontraron discrepancias semánticas. El instrumento original de Dijistra et al. (2010) contiene 38 ítems que se responden indicando qué tan celosa se sentiría la persona ante diversas situaciones en una escala Likert de 1 (nada celoso) a 5 (muy celoso). Los reactivos se agrupan en cuatro dimensiones que reflejan distintas conductas extradiádicas de la pareja, incorporando los medios de comunicación moderna como Internet y mensajes de texto. Las dimensiones descritas por Dijistra et al. (2010) son: (1) Comportamiento infiel, refleja la ocurrencia de infidelidad certera por parte de la pareja (como tener una cita romántica, enamorarse, o tener relaciones sexuales con otra persona); (2) Comportamiento sospechoso, implica conductas que no son explícitamente situaciones de infidelidad, pero que sí podrían conllevar a esta, como la pareja bailando con otro, teniendo una conversación interesante o abrazando a otra persona; (3) Pornografía, se refiere a la infidelidad relacionada al consumo de material pornográfico, como la pareja mirando este tipo de material en Internet o viendo una película pornográfica; (4) Inversión tecnológica, involucra el que la pareja desarrolle intimidad emocional con otra persona a través de medios tecnológicos, como la pareja compartiendo sentimientos y secretos por chat o correo electrónico, o intercambiar regularmente mensajes de texto con alguien del sexo opuesto. Los resultados descriptivos y la fiabilidad de las dimensiones del ICPC para la muestra adulta que evaluó Dijistra et al. (2010) se presentan en la tabla 1.

\section{Análisis de datos}

Todos los análisis fueron realizados con el paquete estadístico SPSS 21.0. Se realizó un AFE con extracción de ejes principales y rotación oblimin, comparando la solución con un análisis paralelo de Horn (cf. Matsunaga, 2011), para determinar el número de factores a retener.

Se calcularon estadísticos descriptivos como la media y frecuencias para caracterizar a la muestra en cada dimensión del ICPC, obteniendo la puntuación promedio por dimensión. Se calculó la consistencia interna y el coeficiente de correlación de Pearson para calcular las correlaciones entre los distintos momentos de aplicación del inventario y la asociación entre sus dimensiones. También se utilizaron análisis de varianza múltiple (MANOVA) con las dimensiones del ICPC como variables dependientes y las variables sociodemográficas como variable de agrupación, a fin de determinar cuáles de estas son las que discriminan en las dimensiones del instrumento.

Tabla 1

Resultados descriptivos para las dimensiones del ICPC en el estudio original

\begin{tabular}{lrccc}
\hline & $n i$ & $\begin{array}{c}\text { Hombres } \\
M(D E)\end{array}$ & $\begin{array}{c}\text { Mujeres } \\
M(D E)\end{array}$ & $\begin{array}{c}\alpha \text { de } \\
\text { Cronbach }\end{array}$ \\
\hline Comportamiento sospechoso & 15 & $1.94(0.76)$ & $2.30(0.87)$ & .96 \\
Comportamiento infiel & 14 & $3.89(1.02)$ & $4.19(0.97)$ & .93 \\
Pornografía & 5 & $2.12(0.99)$ & $2.53(1.22)$ & .89 \\
Inversión tecnológica & 4 & $2.94(1.16)$ & $3.55(1.20)$ & .89 \\
\hline
\end{tabular}

Nota: $n i=$ número de ítems. Adaptado de Dijistra et al., 2010. 


\section{Resultados}

\section{Análisis de la estructura del ICPC}

Se efectuó un primer AFE con los 38 ítems del ICPC y análisis paralelo, el cual arrojó un total de tres factores a retener, con el que se pudo detectar una alta multicolinearidad en dos ítems con cargas factoriales estandarizadas sobre 1 (casos Heywood) en el primer factor (i.e., "Su pareja tiene sexo con otra persona" y "Su pareja tiene sexo oral con otra persona"). Al evaluar su contenido, se observó que aludían a aspectos muy similares a los evaluados por los primeros dos ítems del instrumento (la pareja tiene una aventura sexual y tiene sexo sin coito, respectivamente), por lo tanto se optó por eliminar los dos ítems problemáticos, siguiendo ambos criterios, el estadístico y el sustantivo. De este modo, se llevó a cabo un segundo AFE con los 36 ítems restantes y de acuerdo con el nuevo análisis paralelo se volvió a seleccionar tres factores, llegando a una solución que explicó un 63.97\% de la varianza, $\mathrm{KMO}=.943, \chi^{2}(630)=$ 11197.99, $p<.001$. Asimismo, en el segundo AFE hubo 10 ítems que resultaron eliminados debido a que no cargaron en los factores a retener, así como un ítem que presentó una carga factorial estandarizada inferior a .4 (LloretSegura, Ferreres-Traver, Hernández-Baeza y Tomás-Marco, 2014), llegando a una solución que explicó un $63.97 \%$ de la varianza, $\mathrm{KMO}=$ $.943, \chi^{2}(630)=11197.99, p<.001$.

Al realizar un análisis del contenido de las dimensiones seleccionadas y los ítems que agrupó la versión chilena del ICPC, se pudo observar diferencias respecto a la conceptualización de las dimensiones y algunos de los reactivos que conformaron cada categoría, y la eliminación final de un ítem ("Su pareja idolatra a una persona famosa del sexo opuesto") por presentar una carga factorial inferior a 4 (Lloret-Segura et al., 2014).

El resultado anterior implicó llegar a una solución definitiva con tres factores y 25 ítems, es decir, un factor y 13 ítems menos de lo que reporta Diijstra et al., 2010 (quienes encuentran un $59.42 \%$ de varianza explicada en estudiantes y $67.99 \%$ en una muestra de adultos). A su vez, luego de la rotación de los factores, se observó una correlación de .45 entre el primer y segundo factor, de .32 entre el primer y el tercer factor, y de .41 entre el segundo y el tercer factor. Los resultados del análisis paralelo en contraste con los observados en la muestra para la presente aplicación se muestran en la tabla 2.

Las dimensiones de la versión chilena del ICPC contemplaron un primer factor con 12 ítems denominado Comportamiento infiel, que agrupa situaciones explicitas de infidelidad (tener una aventura sexual, besar a otra persona, tener una cita romántica, tener sexo por Internet o por teléfono, coquetear con otra persona). El segundo factor mantuvo el sentido original de Comportamiento sospechoso, agrupando ocho ítems referidos a situaciones que podrían generar suspicacias (que la pareja tenga una vida social independiente y relaciones cercanas con alguien del sexo opuesto, como bailar o abrazar a alguien del sexo opuesto, trabajar o pasar mucho tiempo con alguien del sexo opuesto), pero sin la ocurrencia de infidelidad explícita.

Tabla 2

Autovalores y varianza explicada inesperados y observados en el primer análisis factorial exploratorio

\begin{tabular}{|c|c|c|c|c|c|c|}
\hline & \multicolumn{3}{|c|}{ Primer análisis (38 ítems) } & \multicolumn{3}{|c|}{ Análisis final (25 ítems) } \\
\hline & \multirow{2}{*}{$\begin{array}{c}\text { AP } \\
\begin{array}{c}\text { Autovalor } \\
\text { esperado }\end{array}\end{array}$} & \multicolumn{2}{|c|}{$\mathrm{AFE}$} & \multirow{2}{*}{$\begin{array}{c}\text { AP } \\
\begin{array}{c}\text { Autovalor } \\
\text { esperado }\end{array}\end{array}$} & \multicolumn{2}{|c|}{ AFE } \\
\hline & & $\begin{array}{l}\text { Autovalor } \\
\text { observado }\end{array}$ & $\begin{array}{r}\text { Varianza } \\
\text { explicada }\end{array}$ & & $\begin{array}{l}\text { Autovalor } \\
\text { observado }\end{array}$ & $\begin{array}{r}\text { Varianza } \\
\text { explicada }\end{array}$ \\
\hline F1 & 1.72 & 16.03 & $42.19 \%$ & 1.70 & 15.08 & $41.88 \%$ \\
\hline $\mathrm{F} 2$ & 1.64 & 5.44 & $14.33 \%$ & 1.61 & 4.92 & $13.67 \%$ \\
\hline F3 & 1.57 & 3.04 & $7.99 \%$ & 1.54 & 3.04 & $8.43 \%$ \\
\hline
\end{tabular}

Nota AP = Análisis Paralelo; AFE = Análisis Factorial Exploratorio. Los autovalores del análisis paralelo corresponden a valores esperados por azar. 
El tercer factor, incorporó ítems relacionados a Atracción mediática quedando constituido por cinco elementos (ver pornografía a través de películas, mirar con interés a alguien del sexo opuesto en la TV). En la tabla 3 se muestran las cargas factoriales de la configuración final del inventario en su adaptación para la población chilena.

Tabla 3

Cargas factoriales de la estructura factorial definitiva del ICPC

\begin{tabular}{|c|c|c|c|c|}
\hline \multirow[b]{2}{*}{ Comportamiento de la pareja } & \multicolumn{3}{|c|}{ FACTOR } & \multirow{2}{*}{ Comunalidad } \\
\hline & I & II & III & \\
\hline $\begin{array}{l}\text { 1. Su pareja tiene una aventura sexual con otra } \\
\text { persona }\end{array}$ & .899 & .097 & -.042 & .884 \\
\hline $\begin{array}{l}\text { 2. Su pareja tiene sexo con otra persona, sin } \\
\text { tener coito }\end{array}$ & .945 & .077 & -.018 & .915 \\
\hline $\begin{array}{l}\text { 3. Su pareja se desviste para otra persona frente } \\
\text { a la cámara del computador }\end{array}$ & .909 & .086 & .049 & .734 \\
\hline 4. Su pareja besa a otra persona & .838 & .062 & .029 & .761 \\
\hline $\begin{array}{l}\text { 5. Su pareja envía mensajes o correos electró- } \\
\text { nicos de índole sexual a alguien del sexo opues- } \\
\text { to }\end{array}$ & .920 & .032 & .023 & .860 \\
\hline $\begin{array}{l}\text { 6. Su pareja tiene una cita romántica con otra } \\
\text { persona }\end{array}$ & .719 & -.066 & -.019 & .791 \\
\hline $\begin{array}{l}\text { 7. Su pareja se enamora de alguien con quien se } \\
\text { comunica a través de Internet }\end{array}$ & .632 & -.103 & .014 & .810 \\
\hline $\begin{array}{l}\text { 8. Su pareja tiene sexo telefónico con otra per- } \\
\text { sona }\end{array}$ & .883 & -.024 & .056 & .812 \\
\hline $\begin{array}{l}\text { 9. Su pareja envía una carta romántica o postal } \\
\text { a alguien del sexo opuesto }\end{array}$ & .758 & -.007 & -.009 & .778 \\
\hline 10. Su pareja es atraída por otra persona & .432 & -.024 & .120 & .697 \\
\hline $\begin{array}{l}\text { 11. Su pareja tiene sexo mediante Internet con } \\
\text { otra persona }\end{array}$ & .908 & -.003 & .060 & .822 \\
\hline 12. Su pareja baila con alguien del sexo opuesto & .068 & .506 & .019 & .694 \\
\hline 13. Su pareja abraza a alguien del sexo opuesto & .035 & .595 & -.003 & .679 \\
\hline $\begin{array}{l}\text { 14. Su pareja trabaja intensamente con alguien } \\
\text { del sexo opuesto }\end{array}$ & .054 & .660 & -.031 & .645 \\
\hline $\begin{array}{l}\text { 15. Su pareja llama por teléfono a alguien del } \\
\text { sexo opuesto }\end{array}$ & .081 & .812 & -.027 & .600 \\
\hline $\begin{array}{l}\text { 16. Su pareja pasa mucho de su tiempo con } \\
\text { alguien del sexo opuesto }\end{array}$ & .179 & .790 & -.067 & .573 \\
\hline $\begin{array}{l}\text { 17. Su pareja mira con interés a un individuo } \\
\text { del sexo opuesto en la TV }\end{array}$ & -.001 & .440 & .500 & .553 \\
\hline 18. Su pareja sale sin Ud. & -.063 & .590 & .177 & .493 \\
\hline $\begin{array}{l}\text { 19. Su pareja da un regalo a alguien del sexo } \\
\text { opuesto }\end{array}$ & .036 & .559 & .071 & .562 \\
\hline $\begin{array}{l}\text { 20. Su pareja le cuenta cuán atractiva piensa } \\
\text { que es una persona del sexo opuesto }\end{array}$ & .142 & .453 & .088 & .517 \\
\hline
\end{tabular}


Tabla 3

Cargas factoriales de la estructura factorial definitiva del ICPC (continuación)

\begin{tabular}{lllll} 
21. Su pareja coquetea con alguien más &. $\mathbf{4 8 0}$ & .038 & .099 & .659 \\
$\begin{array}{l}\text { 22. Su pareja ve una película pornográfica sin } \\
\text { Ud. }\end{array}$ & .094 & -.089 & $\mathbf{. 9 4 3}$ & .808 \\
$\begin{array}{l}\text { 23. Su pareja mira fotografías o películas porno- } \\
\text { gráficas en Internet }\end{array}$ & .049 & -.070 & $\mathbf{. 9 8 0}$ & .933 \\
$\begin{array}{l}\text { 24. Su pareja mira fotografías pornográficas en } \\
\text { una revista }\end{array}$ & .052 & -.090 & $\mathbf{. 9 6 4}$ & .902 \\
$\begin{array}{l}\text { 25. Su pareja mira fotografías (no pornográficas) } \\
\text { de un modelo atractivo/a del sexo opuesto en } \\
\text { una revista }\end{array}$ & -.098 & .153 & $\mathbf{. 7 5 5}$ & .707 \\
\hline
\end{tabular}

Nota: Los valores en negrita representan la carga de cada ítem que pertenece a cada uno de los factores retenidos. El Factor I representa los ítems en la dimensión Comportamiento infiel. El Factor II agrupa los ítems de la dimensión Comportamiento sospechoso. El Factor III se denomina Atracción mediática.

\section{Fiabilidad}

Se calculó la fiabilidad de la escala por consistencia interna ( $\alpha$ de Cronbach) para cada una de las dimensiones del inventario. Luego se procedió a calcular el coeficiente de correlación $r$ de Pearson, como indicador de la estabilidad del instrumento entre la primera y la segunda aplicación (test-retest para un lapso de 60 días). Para dicho análisis se consideraron todas las personas $(n=$ 31) que accedieron a responder el ICPC en una segunda ocasión (ver tabla 4). Cabe notar que para este lapso de 60 días, los valores promedio alcanzados en cada dimensión no se diferenciaron significativamente de la media por dimensión para la segunda aplicación ( $d$ de Cohen entre aplicaciones de 0.01 a 0.09 ).

\section{Contraste de las hipótesis teóricas con res- pecto al ICPC}

En conformidad con los postulados evolucionarios, se constató que las mujeres obtenían valores significativamente más altos que los hombres en dos de las tres dimensiones del ICPC mediante MANOVA (ver tabla 5).

Finalmente, se evaluó mediante MANOVA si existían diferencias en las dimensiones de la escala según si las personas se encontraban saliendo exclusivamente con alguien o no. Los resultados mostraron que aquellas personas que estaban actualmente saliendo con una persona exclusivamente, obtenían puntuaciones significativamente más altas que quienes no en la dimensión de Atracción mediática (ver tabla 6).

Tabla 4

$V$ alores descriptivos y fiabilidad de las dimensiones del ICPC

\begin{tabular}{lccc}
\hline & $\begin{array}{c}\text { Comportamiento } \\
\text { infiel }\end{array}$ & $\begin{array}{c}\text { Comportamiento } \\
\text { sospechoso }\end{array}$ & $\begin{array}{c}\text { Atracción } \\
\text { mediática }\end{array}$ \\
\hline$M(D E)$ & $4.34(0.87)$ & $2.10(0.85)$ & $1.70(0.92)$ \\
$\alpha$ de Cronbach & .97 & .91 & .92 \\
Test-retest $(r)$ & $.77^{*}$ & $.88^{*}$ & $.72^{*}$ \\
\hline
\end{tabular}

Nota: $* p<.001$. 
Tabla 5

Puntuación promedio en cada dimensión del ICPC según sexo

\begin{tabular}{lcccc}
\hline & $\begin{array}{c}\text { Hombre }(n=90) \\
M(D E)\end{array}$ & $\begin{array}{c}\text { Mujer }(n=230) \\
M(D E)\end{array}$ & $F$ & $\eta_{p}^{2}$ \\
\hline Comportamiento infiel & $4.05(1.00)$ & $4.45(0.78)$ & $14.61^{*}$ & .04 \\
$\begin{array}{l}\text { Comportamiento } \\
\text { sospechoso }\end{array}$ & $1.82(0.72)$ & $2.18(0.88)$ & $11.51^{*}$ & .04 \\
Atracción mediática & $1.59(0.83)$ & $1.74(0.95)$ & 1.74 & .01 \\
\hline
\end{tabular}

Nota: F = Estadístico F de Fisher-Snedecor. Los grados de libertad del numerador y denominador para las tres pruebas fueron 1 y 319 respectivamente.

$* p<.001$.

\section{Discusión}

El objetivo del estudio fue adaptar al contexto chileno el ICPC. El inventario fue traducido y aplicado a una muestra de hombres y mujeres, encontrándose una configuración cercana a la conceptualización original en Holanda, pero con particularidades propias del contexto local, que hicieron disminuir un factor y eliminar diversos ítems. La consistencia interna de las dimensiones de la escala fue adecuada y su estabilidad temporal fue moderada.

El AFE permitió establecer las dimensiones en las cuales se separan las situaciones de celos, quedando el inventario conformado por 25 ítems. La dimensión de Comportamiento infiel incorporó todas las situaciones claras de infidelidad por parte de una pareja, integrando situaciones de infidelidad sexual, incorporando además el coqueteo que no estaba contemplado en el estudio original (Diijstra et al., 2010). Asimismo, el Comportamiento sospechoso mantuvo sus características originales, pero el mirar con interés a alguien en la TV pasó a ser parte del tercer factor. Finalmente, Atracción mediática agrupó conductas de consumo de pornografía en fotografías y películas, así como la atracción hacia personas que aparecen en los medios o son famosas. De esta forma, en el presente estudio la dimensión de Dijistra et al., (2010) referente a la pornografía se elimina del instrumento chileno, quedando algunos de sus elementos incluidos en la Atracción mediática por personas famosas, sean estos pornográficos o medios de comunicación comunes.

Una importante limitación de la aplicación chilena del ICPC concierne a la incorporación de la apli- cación por Internet del instrumento, lo cual podría haber afectado la falta de convergencia de la estructura original en el contexto local. Por ejemplo, Weigold, Weigold y Russell (2013) han mostrado que existe una mayor tasa de datos perdidos, menos ítems sin responder y mayor tiempo de respuesta en los instrumentos aplicados por Internet, lo cual podría haber afectado la falta de ajuste del modelo confirmatorio del ICPC en el contexto local, además de posibilitar una constitución de muestra de tipo accidental, cuyos resultados pueden ser más difíciles de generalizar. De igual forma, otra posible desventaja acerca de la representatividad de la muestra fue la eliminación de dos ítems que cargan sobre uno en un primer AFE que incluyó todos los ítems del instrumento original; si bien Joreskog (1999) reconoce esta situación como un posible indicador de excesiva multicolineariedad, señala que no afecta la calidad global del AFE. No obstante, en el presente estudio se optó por eliminar del primer factor los ítems problemáticos siguiendo adicionalmente un criterio sustantivo, por la referencia excesiva a situaciones de infidelidad sexual y sin coito.

Por otra parte, teóricamente hablando, es interesante que se pudo constatar que, tal como lo predice la psicología evolucionaria, se observan diferencias sexuales en los celos pese a la falta de generalización de la muestra, siendo para las mujeres más altos los niveles de reactividad a los celos en las dos primeras dimensiones evaluadas. Esto se debería posiblemente a que ellas son quienes más pueden perder la alta inversión en una relación reproductiva al perder su pareja ante un rival (para una discusión del valor adaptativo de esta diferencia, cf. Buss, 2013b). 
Tabla 6

Puntuación promedio en cada dimensión según nivel de compromiso

\begin{tabular}{lcccc}
\hline & $\begin{array}{l}\text { Sale } \\
\text { exclusivamente } \\
(n=89)\end{array}$ & $\begin{array}{l}\text { No sale } \\
\text { exclusivamente } \\
(n=214) \\
M(D E)\end{array}$ & $F$ & $\eta_{p}^{2}$ \\
& $4.38(0.84)$ & $4.23(0.97)$ & 1.87 & .01 \\
\hline $\begin{array}{l}\text { Comportamiento infiel } \\
\text { Comportamiento }\end{array}$ & $2.12(0.89)$ & $1.99(0.77)$ & 1.40 & .01 \\
$\begin{array}{l}\text { sospechoso } \\
\text { Atracción mediática }\end{array}$ & $1.80(0.98)$ & $1.45(0.72)$ & $9.45^{*}$ & .03 \\
\hline
\end{tabular}

Nota: F = Estadístico F de Fisher-Snedecor. Los grados de libertad del numerador y denominador para las tres pruebas fueron 1 y 302 respectivamente.

$* p<.01$.

Tal como lo ha planteado Dijkstra et al. (2010), las situaciones que evocan celos dentro de una díada romántica son muchas veces esperables dentro de cualquier relación y no necesariamente ausentes en las relaciones de pareja saludables, mostrando esta primera evaluación de los comportamientos que provocan celos en nuestro país una mayor reactividad femenina, a excepción de la Atracción mediática.

Igualmente, a diferencia de la popular consideración de los celos como una conducta patológica por parte de una pareja, en el estudio se puede observar que si bien esta conducta no se diferencia fuertemente en quienes mantienen una relación de pareja en la actualidad, el salir exclusivamente con una persona conlleva a una mayor reacción de celos en la dimensión de Atracción mediática, lo que da cuenta de la existencia de una valoración de la relación por parte de quienes apuestan por la exclusividad, reaccionando a estas situaciones de baja certeza de ocurrencia de infidelidad con mayores niveles de celos, posiblemente debido a la expectativa de que la exclusividad debiese alejar a la pareja de otras personas atractivas o de la pornografía (Dijkstra et al., 2010).

En conclusión, la presente adaptación del Inventario de Comportamientos de la Pareja que Provocan Celos permite contar con una herramienta útil para continuar explorando en la investigación en el contexto local. Pese a que la validación se realizó con una muestra con una gran cantidad de mujeres en comparación con hombres, puede de todas formas facilitar el entendimiento de las respuestas que frecuentemente sienten las parejas dentro de una relación valorada y ayudar a la disciplina psicológica a identificar situaciones y patrones de comportamiento que hacen emerger estos sentimientos. Queda a futuras investigaciones con el instrumento indagar en otras especificidades y diferencias sexuales en los comportamientos que evocan los celos en una relación de pareja, idealmente contando con muestras equiparadas por sexo y comparando la prevalencia de dichas conductas en diversidad de personas con y sin un vínculo relacional actual, así como en población clínica o personas con antecedentes de celotipia.

\section{Referencias}

Barkow, J. H., Cosmides, L. y Tooby, J. (1992). The adapted mind: Evolutionary Psychology and the generation of culture. New York: Oxford University Press.

Buss, D. M. (1989). Conflict between the sexes: Strategic interference and the evocation of anger and upset. Journal of Personality \& Social Psychology, 56(5), 735-747.

http://dx.doi.org/10.1037//0022-

3514.56.5.735

Buss, D. M. (1995). Psychological sex differences: Origins through sexual selection. 
American Psychologist, 50(3), 164-168.

http://dx.doi.org/10.1037//0003-066X.50.3.164

Buss, D. M. (2005). The handbook of Evolutionary Psychology. Nueva York: Wiley.

Buss, D. M. (2009). The great struggles of life: Darwin and the emergence of Evolutionary Psychology. American Psychologist, 64(2), 140148.

http:/ /dx.doi.org/10.1037/a0013207

Buss, D. M. (2011). Evolutionary Psychology. The new science of the mind. Boston: Pearson.

Buss, D. M. (2013a). The science of human mating strategies: An historical perspective. Psychological Inquiry: An International Journal for the Advancement of Psychological Theory, 24(3), 171-177.

http://dx.doi.org/10.1080/1047840X.2013. 819552

Buss, D. M. (2013b). Sexual jealousy. Psychological Topics, 22, 155-182.

Buss, D. M., Larsen, R. J., Westen, D. y Semmelroth, J. (1992). Sex differences in jealousy: Evolution, physiology, and psychology. Psychological Science, 3(4), 251-255. http://dx.doi.org/10.1111/j.14679280.1992.tb00038.x

Buss, D. M., Shackelford, T. K., Kirkpatrick, L.A., Choe, J.C., Lim, H.K., Hasegawa, M., ..., Bennett, K. (1999). Jealousy and the nature of beliefs about infidelity: Tests of competing hypotheses about sex differences in the United States, Korea, and Japan. Personal Relationships, 6(1), 125-150. http://dx.doi.org/10.1111/j.14756811.1999.tb00215.x

Buunk, A. P. (1997). Personality, birth order and attachment styles as related to various types of jealousy. Personality and Individual Differences, 23(6), 997-1006.

http://dx.doi.org/10.1016/S0191-8869(97)00136-0

Buunk, A., Park, J. H., Zurriaga, R., Klavina, L. y Massar, K. (2008). Height predicts jealousy differently for men and women. Evolution and
Human Behavior 29(2), 133-139.

http://dx.doi.org/10.1016/j.evolhumbehav.200 7.11 .006

Buunk, B. P. y Dijkstra, P. (2001). Evidence from a homosexual sample for a sex-specific rival-oriented mechanism: Jealousy as a function of a rival's physical attractiveness and dominance. Personal Relationships, 8(4), 391-406.

http://dx.doi.org/10.1111/j.1475-

6811.2001.tb00047.x

Cosmides, L. y Tooby, J. (1992). Cognitive adaptations for social exchange. En J. Barkow, L. Cosmides y J. Tooby (Eds.), The adapted mind: Evolutionary Psychology and the generation of culture (pp. 163-228). Nueva York: Oxford University Press.

Cosmides, L. y Tooby, J. (1999). Evolutionary Psychology. En R. A. Wilson y F. C. Keil (Eds), MIT Encyclopedia of Cognitive Science (pp. 294-297). Cambridge, MA: MIT Press.

Cosmides, L. y Tooby, J. (2013). Evolutionary Psychology: New perspectives on cognition and motivation. Annual Review of Psychology, 64(1), 201-229.

http://dx.doi.org/10.1146/annurev.psych.12 1208.131628

De Miguel, A. y Buss, D. M. (2011). Mate retention tactics in Spain: Personality, sex differences, and relationship status. Journal of Personality, 79(3), 563-586.

http://dx.doi.org/10.1111/j.14676494.2011.00698.x

Darwin, C. (1859). The origin of species. Nueva York: Mentor.

Dijkstra, P., Barelds, D. P. H. y Groothof, H. A. K. (2010). An inventory and update of jealously-evoking partner behaviors in modern society. Clinical Psychology and Psychoterapy, 17, 329-345. http://dx.doi.org/10.1002/cpp.668

Dijkstra, P. y Buunk, B. P. (2002). Sex differences in jealousy-evoking effects of rival characteristics. European Journal of Social 
Psychology, 32(6), 829-852.

http://dx.doi.org/10.1002/ejsp.125

Dunbar, R. (2012). The Science of Love and Betrayal. Nueva York: Wiley.

Elphinston, R. A., Feeney, J. A. y Noller, P. (2011). Measuring romantic jealousy: Validation of the multidimensional jealousy scale in Australian samples. Australian Journal of Psychology, 63 (4), 243-251.

http://dx.doi.org/10.1111/j.17429536.2011.00026.x

Fernández, A. M. (2009). Psicología evolucionaria: un marco integrador para la disciplina psicológica en el bicentenario de Darwin. Revista de Psicología Universidad de Chile, 18, 9-24.

Fernández, A. M. (2012). Psicofisiología de los celos románticos: estudio experimental de las emociones que surgen ante la infidelidad desde la perspectiva evolucionaria En F. Barrera (Ed.), Tesis doctorales en Psicologia: Compendio 2011 (pp. 71-105). Santiago: Lom.

Fisher, H. (1996). Anatomy of love: A natural history of mating, marriage, and why we stray. Nueva York: W. W. Norton.

Hernández, R., Fernández-Collado, C. y Baptista, P. (2010). Metodología de la investigación. Ciudad de México: McGraw-Hill.

Joreskog, K. G. (1999). How large can a standardized coefficient be? Manuscrito inédito. Recuperado de https://is.gd/JM2vt3

Lloret-Segura, S., Ferreres-Traver, A., Hernández-Baeza, A. y Tomás-Marco, I. (2014). El análisis factorial exploratorio de los ítems: una guía práctica, revisada y actualizada. Anales de
Psicología, 30, 1151-1169.

http://dx.doi.org/10.6018/analesps.30.3.199361

Maner, J. K. y Shackelford, T. K. (2008). The basic cognition of jealousy: An evolutionary perspective. European Journal of Personality, 22(1), 31-36.

http://dx.doi.org/10.1002/per.661

Matsunaga, R. (2011). How to factor analyze your data right: Do's, don'ts and how-to's. International Journal of Psychological Research, 3, 97-110.

Muñiz, J., Elosua, P. y Hambleton, R. K. (2013). Directrices para la traducción y adaptación de los test: segunda edición. Psicothema, 25, 151-157. http://dx.doi.org/1.7334/psicothema2013.24

Nunnally, J. C. y Bernstein, I. H. (1994). Psychometric theory. Nueva York: McGraw-Hill.

Sznycer, D., Tooby, J. y Cosmides, L. (2011). Evolutionary Psychology. Recuperado de http://www.cep.ucsb.edu/papers/2011Encyclopedia_EvPsych.pdf

Triandis, H. C. y Berry, J. W. (Eds.) (1980). Handbook of cross-cultural psychology: Methodology (Vol. 2). Boston: Allyn \& Bacon.

Watkins, M. (2008). Monte Carlo for PCA parallel analysis (version 2.3). Recuperado de http://www.softpedia.com/get/Others/Ho me-Education/Monte-Carlo-PCA-forParallel-Analysis.shtml

Weigold, A., Weigold, I. K. y Russell, E. J. (2013). Examination of the equivalence of self-report survey-based paper-and-pencil and internet data collection methods. Psychological Methods, 18(1), 53-70. http://dx.doi.org/10.1037/a0031607

Fecha de recepción: 30 de marzo de 2014 Fecha de aceptación: 3 de septiembre de 2014

Corrección: Este artículo fue publicado originalmente con un error en la tabla 3. En dicha versión, se marcaron los números .038, -.089, -.070, -.090 y .153 con negrita; lo cuales corresponden al factor II de los ítems 21, 22, 23, 24 y 25. En esta versión (de junio de 2016) este error se ha corregido, marcando con negrita los números .480, del factor I, y .943, .980, .964 y .755, del factor 3, de los mismos ítems. 